\title{
TWO NEW PEDESTRIAN NAVIGATION PATH OPTIONS BASED ON SEMI-INDOOR SPACE
}

\author{
J. Yan ${ }^{1, *}$, S. Zlatanova ${ }^{1}$, A. A. Diakite ${ }^{1}$ \\ ${ }^{1}$ UNSW Built Environment, Red Centre Building, Kensington NSW 2052, Sydney, Australia \\ (jinjin.yan, s.zlatanova, a.diakite)@unsw.edu.au
}

Commission IV, WG IV/10

KEY WORDS: Pedestrian Navigation, Semi-indoor Space, MTC-path, NSI-path, Shortest Path

\begin{abstract}
:
Navigation is very critical for our daily life, especially when we have to go through the unfamiliar areas where the spaces are very complex, such as completely bounded (indoor), partially bounded (semi-indoor and/or semi-outdoor), entirely open (outdoor), or combined. Current navigation systems commonly offer the shortest distance/time path, but it is not always appropriate for some situations. For instance, on a rainy day, a path with as many places that are covered by roofs/shelters is more attractive. However, current navigation systems cannot provide such kinds of navigation paths, which can be explained by that they lack information about such roofed/sheltered-covered spaces. This paper proposes two roofed/sheltered navigation path options by employing semi-indoor spaces in the navigation map: (i) the Most-Top-Covered path (MTC-path) and (ii) path to the Nearest sI-space from departure (NSI-path). A path selection strategy is introduced to help pedestrians in making choices between the two new path options and the traditional shortest path. We demonstrate and validate the research with path planning on two navigation cases. The results show the two path options and the path selection strategy bring in new navigation experience for humans.
\end{abstract}

\section{INTRODUCTION}

Navigation is important for agents when they have to go through the unfamiliar and complex living areas (environments). Here, agents are clients that use or engage in navigation activities. They could be humans or human proxies, such as robots or drones programmed by humans. In this paper, we concentrate on humans. Based on transportation modes, agents are classified as pedestrians, people with mobility constraints (wheeled users, drivers), and cyclists. The structure of complex living areas (environments) are broadly subdivided into four types: indoor (I-space), semi-indoor (sI-space), semi-outdoor (sO-space), and outdoor (O-space) (Yan et al., 2020, Yan et al., 2019a, Yan et al., 2019b).

When it refers to navigation path options, the shortest distance/time paths are generally the only options in the available navigation systems. That is, distance/time is the only criterion of path options. The two kinds of options could be a habitual choice for humans, but it does not mean they are always expecting choices under all circumstances. For instance, a least-top-exposed path (Yan et al., 2019b) can be more interesting and attractive on a rainy day, since roofs/shelters of the semi-indoor spaces can offer protection for people to escape from the rain.

In this research, the concept of sI-space corresponds with the definitions in the previous research (Yan et al., 2019b). The sI-spaces are the hollow parts formed by living environments that are semi-open to the outdoors, physically enclosed by top(s) (e.g., roof, shelter), and may have a side(s) (e.g., wall, fence), but is not physically enclosed completely like indoor. Furthermore, this paper continues to use the term "top closure $\left(C^{T}\right)$ " introduced in (Yan et al., 2019a, Yan et al., 2019b), which is utilized for defining sI-spaces. The $C^{T}$ is a coefficient

\footnotetext{
* Corresponding author
}

that expresses how much a space is physically bounded from its top. It corresponds to the ratio between the substantial (material) area and the entire area of the top boundary structure.

This paper proposes two new path options based on semi-indoor spaces. From the characteristics of the spaces, sI-spaces and I-spaces are two types of spaces that have tops, but this research considers the sI-spaces only, because the sI-spaces are mostly public (Zhu et al., 2016) while the I-spaces are often regarded as non-public. The two path options are (i) the Most-Top-Covered path (MTC-path) and (ii) path to the Nearest sI-space from departure (NSI-path). The MTC-path is similar to the least-top-exposed path (proposed by (Yan et al., 2018, Yan et al., 2019b)) from notion point of view, but it differs from two aspects: the MTC-path is a parameters-based path option and it takes both travel distance and the top-coverage-ratio (see definition in Section 3.1.2) as the criteria in path determination. The NSI-path is proposed to help pedestrians to find a closest roofed/sheltered place from their departures, which is a compromise option when neither the shortest path nor MTC-path is not recommended (see the path selection strategy in Section 3.4).

The remaining sections are organized as follows. Section 2 explains the current research on the navigation path options. Section 3 introduces the two path options and a path selection strategy. Section 4 presents the implementations for demonstrating and validating our approach, upon which conclusions and future work are drawn in the final section.

\section{RELATED WORK}

Current navigation systems primarily take the travel distance (shortest) or time (fastest) as the main criteria for optimal path computation (Dudas et al., 2009, Ghafourian , Karimi, 2009), although diverse travel modes are considered, such as walking 
(Karimi et al., 2013), driving (Millonig , Schechtner, 2007), transit (Zar, Sein, 2016), and cycling (Howard, Burns, 2001). The path with the shortest distance or fastest travel time could be a habitual choice for humans (Golledge, 1995), but it does not mean this kind of path is always the best choice under all circumstances.

In the past decades, a lot of research have been reported on personalized/agent-tailored navigation paths, such as shortest-distance/time path (Kimmel et al., 1995), simplest (minimum turns) path (Duckham , Kulik, 2003) , least/most-space-visited, least-obstruction (Liu , Zlatanova, 2013), safe path (Andreev et al., 2015, Balata et al., 2018, Cambra et al., 2019, Wang , Zlatanova, 2019), health-optimal routing (e.g., specific level of calories burn) (Sharker et al., 2012), minimum traffic related air pollution exposure (Alam et al., 2018).

\section{MTC-PATH, NSI-PATH \& A PATH SELECTION STRATEGY}

This section introduces the parameters of the navigation model and navigation path. Then, the MTC-path, NSI-path, and a path selection strategy is presented. The traditional shortest path can be the shortest distance or travel time path. In this paper, the shortest path mentioned below refers to the path with the shortest distance. It should be mentioned the navigation model in this paper is a network-based navigation model, which is derived from 3D spaces on the basis of the Poincaré duality (Munkres, 1984). The shortest path is computed based on the Dijkstra algorithm (Dijkstra, 1959).

\subsection{Parameters}

In order to quantitatively reflect the navigation network and navigation path, this section introduces several parameters.

\subsubsection{For Navigation Model}

The parameters for the navigation model are defined based on two connected spaces (Figure 1). Five parameters are defined: the distance between the two connected spaces, original weights, covered \& uncovered distance, uncovered ratio, and modified weights. The definitions and notations of the parameters are the following:

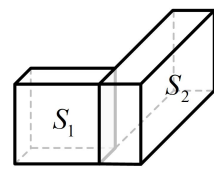

(a)

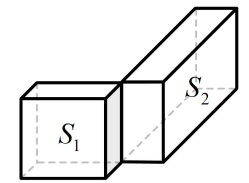

(b)

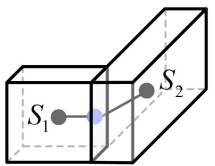

(d)

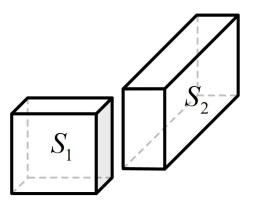

(c)

$$
W_{S_{i j}}^{\prime \prime}=\xi W_{S_{i j}}^{\prime}+(1-\xi) \lambda_{S_{i j}}
$$

where $\xi$ is the coefficient that quantifies the importance of original weights and uncovered ratio, where $\xi \in[0,1]$; $W_{S_{i j}}^{\prime}$ is the original weight; $\lambda_{S_{i j}}$ is the uncovered ratio.

\subsubsection{For Navigation Path}

The parameters for the navigation path are defined based on the planned paths, which are used as quantitative indicators for path comparisons in the later section. The definitions and notations of the parameters are as follows:

in (a) are regarded as connected spaces while that in (b) and (c) are unconnected. The (d) shows the navigation network between the two spaces, in which the blue dot is the additional vertex on the face two spaces touch to indicate how to traverse the spaces.

- The distance between two connected spaces $\left(D_{S_{i j}}\right)$

\section{- Path length $\left(P_{l}\right)$}

The $P_{l}$ is the distance from departure to destination following the planned path. 


$$
P_{l}=\sum D_{s_{i j}}
$$

- Covered/Uncovered length of a path $\left(P_{l_{c}} / P_{l_{u c}}\right)$

Covered length means the total distance of the path segments formed by sI-spaces in the planned path, while the uncovered distance means the total distance of segments formed by sO-space and/or O-space.

$$
P_{l_{c}}=\sum D_{c_{S_{i j}}}, P_{l_{u c}}=\sum D_{u c_{S_{i j}}}
$$

- Top-coverage-ratio of a path $\left(P_{c_{r}}\right)$

The top-coverage-ratio of a path is an indicator that shows how much a path is physically bounded by tops. It is the ratio between the covered length and path length (Equation $6)$.

$$
P_{c_{r}}=P_{l_{c}} / P_{l}
$$

- Weight-based path length $\left(W_{p}\right)$

The weight-based path length is the "length" of a path based on (original/modified) weights. In particular, if a planned path consists of several connected spaces, the $W_{p}$ is the sum of weights corresponding to all the spaces (Equation 7).

$$
W_{p}=\sum W_{S_{i j}}
$$

in which the $W_{S_{i j}}$ is $W_{S_{i j}}^{\prime}$ or $W_{S_{i j}}^{\prime \prime}$.

\subsection{MTC-path}

The MTC-path is a parameters-based path option and it takes both the travel distance and the top-coverage-ratio of a path $\left(P_{c_{r}}\right)$ as the criteria. In short, it aims to determine the shortest path within a top-coverage-ratio constraint in the navigation. The path planning of MTC-path consists of three steps:

- Select sI-spaces. In this step, sI-spaces will be selected based on a threshold of $C^{T}$. This threshold of $C^{T}$ is different from that in the generic space definition framework. Here, it is set on the basis of navigation purposes. For instance, if a pedestrian plans to use the top for escaping from strong sun, the threshold of $C^{T}$ can be set as 0.8 (this value here is only an example). Then, only the sI-spaces with a $C^{T} \geq 0.8$ are selected to participate in the navigation network derivation and navigation path planning. In other words, the results of this step are the sI-spaces that have qualified tops.

- Compute the original and modified weights. Taking the Poincaré duality as the theoretical background, the navigation network is derived based on the selected sI-spaces, sO-spaces, and O-spaces. Then, the original and modified weights are computed based on the Equation 1 and Equation 3 respectively.

- Plan the MTC-path. In this last step, the departure and destination are two nodes in the navigation network corresponding to two spaces and they can be located in semi-indoor, semi-outdoor, or outdoor. After assigning the departure and destination, the modified weights $\left(W_{s_{i} s_{j}}^{\prime \prime}\right)$ are utilized to compute the MTC-path as well as $P_{l}, P_{l_{c}}$, $P_{l_{u c}}$.

\subsection{NSI-path}

Another path option is NSI-path. The process of planning the NSI-path also starts from selecting sI-spaces based on a threshold of $C^{T}$ (the same as that in MTC-path planning). The process of NSI-path planning consists of four steps, which are shown by an illustration (Figure 2). In the example, there are eight selected sI-spaces (sI-1 to sI-8) that are marked by black solid square, $s_{r}$ and $\alpha$ are search radius and search angle respectively. The details of the process are the following:

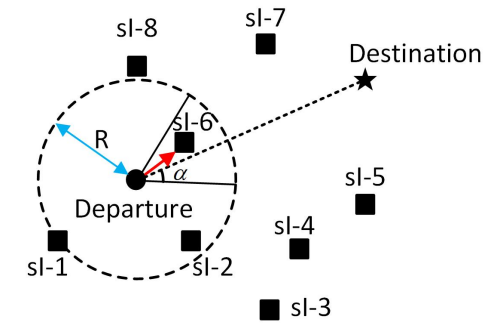

Figure 2. Example of NSI-path planning from departure to destination.

- Create a straight line by linking the departure and destination. This step is to create a line segment by using the location of departure as the start and destination as the end. This line will be used as a reference for the searching area determination.

- Set time $(t)$ and searching angle $(\alpha)$. In this step, two parameters are introduced, time $(t)$ and searching angle $(\alpha)$, in which the former indicates the acceptable time for a pedestrian to move to the nearest sI-space, while the latter is an optimization parameter for determining the preferred search range. The $\alpha$ can vary from 0 to $360^{\circ}$.

- Find potential nearest sI-spaces. With the $t$ and the speed of a pedestrian $(v)$, a search radius $\left(s_{r}\right)$ centred on the departure point can be determined, i.e., $s_{r}=v t$. Then, the searching area becomes a sector by setting a searching angle $\left(\alpha \in\left(0,360^{\circ}\right]\right)$. The searching process is having all the selected sI-spaces to do intersection operations with this sector. If the intersection is not null, the corresponding sI-space will be kept as a candidate for the nearest sI-spaces. With a given $\alpha$, if there is no sI-space within the defined sector, the $\alpha$ will be increased.

- Determine the nearest sI-space and NSI-path. The final step is computing the shortest paths from the departure to each candidate of sI-space based on the Dijkstra algorithm. Then the sI-space corresponding to the path with minimum distance is the nearest sI-space, and this shortest path is the NSI-path. In the demonstration (Figure 2), sI-6 is determined as the nearest sI-space to the departure.

\subsection{A Path Selection Strategy}

More than one paths options can be available and this may create difficulties in selecting a path. It is necessary to have a path selection strategy to make decisions within the traditional shortest path that is currently in use and the two path options. That is, a strategy to help pedestrians to make a choice between three path types: MTC-path, NSI-path, and the traditional shortest path. 
Ideally, pedestrians select the path options with the following order: MTC-path, NSI-path, and the traditional shortest path. However, it is not always sure that a MTC-path is the best option. For example, the length of MTC-path may be longer than the shortest path. Thence, this chapter introduces a path selection strategy (Figure 3) to help pedestrians to find the balance between distance and top-coverage-ratio and set up rules to estimate in which condition which path is the best choice.

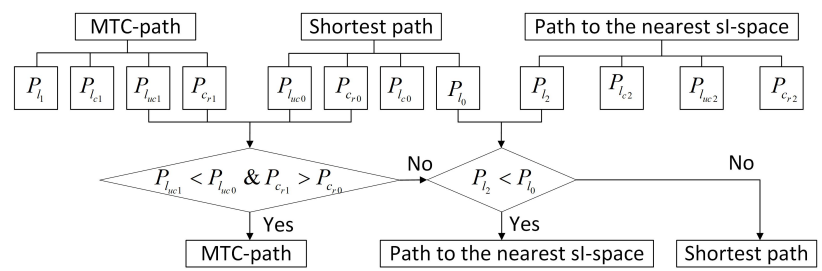

Figure 3. The path selection strategy. The shortest path is computed based on the original weights $\left(W_{S_{i j}}^{\prime}\right)$.

As seen in the path selection strategy, the traditional shortest path is used as the reference, two progressive rules are presented: (i) for the MTC-path, if its uncovered distance is shorter than that of shortest path, and at the same time the top-coverage-ratio is larger than that of shortest path (i.e., $P_{l_{u c 1}}<P_{l_{u c 0}} \& P_{c_{r 1}}>P_{c_{r 0}}$ ), it will be recommended to users preferentially. Otherwise, (ii) the NSI-path will be computed and compared with the traditional shortest path. If the NSI-path is shorter than the shortest path (i.e., $P_{l_{2}}<P_{l_{0}}$ ), the NSI-path will be suggested for pedestrians. Otherwise, the traditional shortest path is the recommendation.

\subsection{Illustration of the Two Path Options}

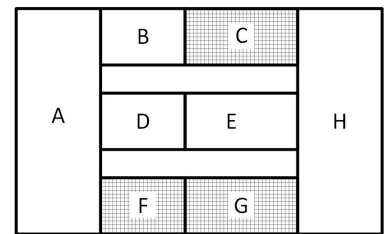

(a)

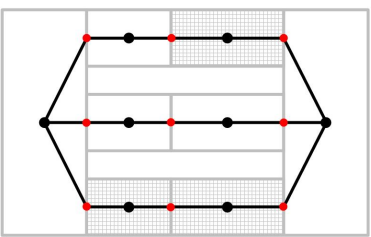

(c)

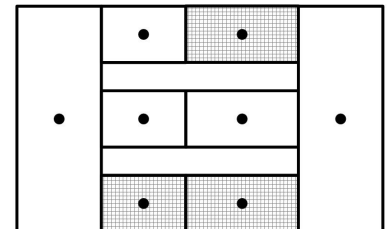

(b)

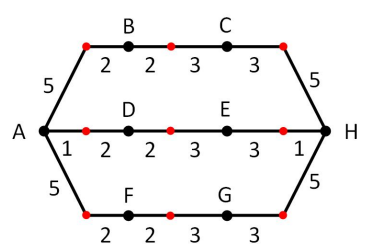

(d)
Figure 4. A navigation example, in which $S_{C}, S_{F}$, and $S_{G}$ are three sI-spaces. (a) All spaces. (b) Nodes extracted from spaces;

(c) Navigation graph derived from spaces based on duality theory, in which the red dots are the extra vertices; (d) Navigation graph with distance.

A navigation example illustrates the two navigation options and the path selection strategy (Figure 4). For simplicity, we use abstract cases containing only spaces that connect by sharing virtual boundaries. There are eight spaces $\left(S_{A}\right.$ to $\left.S_{H}\right)$, in which $S_{C}, S_{F}$, and $S_{G}$ are three selected sI-spaces, while the rests are sO-spaces and O-spaces. Spaces are 3D volumes, but in this example, they are demonstrated in 2D polygons for visualization purposes. Figure $4(a)$ and $(c)$ show the navigation network that derived based on duality theory. The costs of edges are distances, which are identified by numbers without the unit (Figure $4(d)$ ).

With the navigation graph (Figure $4(d)$ ) and distances, original weights (Table 1) are computed based on Equation 1. Then, modified wights $\left(W_{S_{i j}}^{\prime \prime}\right)$ are computed based on Equation 3, in which to show the changes in the modified weights, the coefficient $\xi$ is set from 1 to 0 with intervals of 0.1 (Table 2).

Table 1. Original information of the navigation graph.

\begin{tabular}{ccccccc}
\hline$S_{i}$ & $S_{j}$ & $D_{s_{i j}}$ & $D_{c_{S_{i j}}}$ & $D_{u c_{S_{i j}}}$ & $\lambda_{S_{i j}}$ & $W_{S_{i j}}^{\prime}$ \\
\hline$S_{A}$ & $S_{B}$ & 7 & 0 & 7 & 1 & 0.8 \\
$S_{A}$ & $S_{D}$ & 3 & 0 & 3 & 1 & 0 \\
$S_{A}$ & $S_{F}$ & 7 & 2 & 5 & 0.71 & 0.8 \\
$S_{B}$ & $S_{C}$ & 5 & 3 & 2 & 0.4 & 0.4 \\
$S_{C}$ & $S_{H}$ & 8 & 3 & 5 & 0.625 & 1 \\
$S_{D}$ & $S_{E}$ & 5 & 0 & 5 & 1 & 0.4 \\
$S_{E}$ & $S_{H}$ & 4 & 0 & 4 & 1 & 0.2 \\
$S_{F}$ & $S_{G}$ & 5 & 5 & 0 & 0 & 0.4 \\
$S_{G}$ & $S_{H}$ & 8 & 3 & 5 & 0.625 & 1 \\
\hline
\end{tabular}

With the navigation network and weights, three navigation paths from $S_{A}$ (departure) to $S_{H}$ (destination) are planned (Figure 5), in which path $1\left(S_{A} \rightarrow S_{D} \rightarrow S_{E} \rightarrow S_{H}\right)$ is the traditional shortest path, path $2\left(S_{A} \rightarrow S_{F} \rightarrow S_{G} \rightarrow S_{H}\right)$ is the MTC-path, and path $3\left(S_{A} \rightarrow S_{F}\right)$ is the NSI-path.

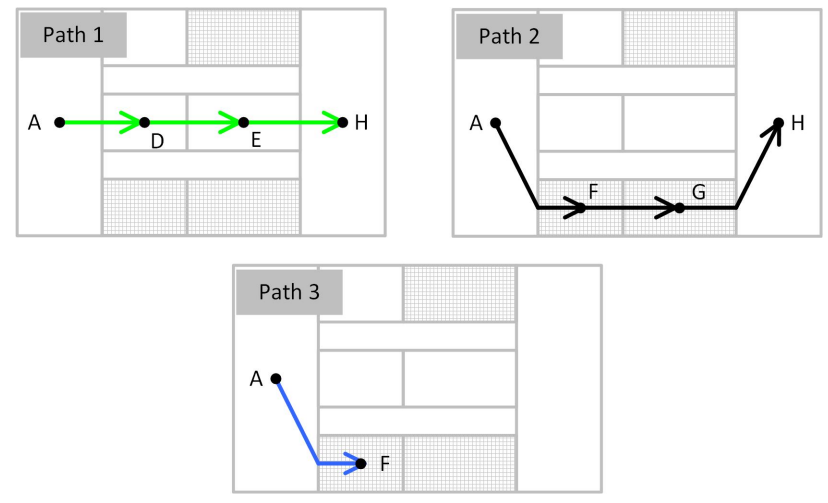

Figure 5. The three navigation paths from $S_{A}$ (departure) to $S_{H}$ (destination). $S_{A} \rightarrow S_{D} \rightarrow S_{E} \rightarrow S_{H}$ is path 1 (green), $S_{A} \rightarrow S_{F} \rightarrow S_{G} \rightarrow S_{H}$ is path 2 (black), and $S_{A} \rightarrow S_{F}$ is path 3 (blue).

The information of the three path options are listed in Table 3. Two show how the information are computed, we select $1-$ $\xi=0.3$ (i.e., $\xi=0.7$ ) as an example. For the path $1, W_{p}=$ $W_{S_{A D}}^{\prime \prime}+W_{S_{D E}}^{\prime \prime}+W_{S_{E H}}^{\prime \prime}=0.3+0.58+0.44=1.32, P_{l}=$ $D_{S_{A D}}+D_{S_{D E}}+D_{S_{E H}}=3+5+4=12, P_{l_{u c}}=D_{u c_{S}}+$ $D_{u c_{S}}+D_{u c_{S_{E}}}=3+5+4=12$, and $P_{c_{r}}=\left(P_{l}-\right.$ $\left.P_{l_{u c}}\right) / P_{l}=(12-12) / 12=0$. Similarly, for the path $2, W_{p}=$ $W_{S_{A F}}^{\prime \prime}+W_{S_{F G}}^{\prime \prime}+W_{S_{G H}}^{\prime \prime}=0.77+0.28+0.89=1.94, P_{l}=$ $D_{S_{A F}}+D_{S_{F G}}+D_{S_{G H}}=7+5+8=20, P_{l_{u c}}=D_{u c_{S}}+$ $D_{u c_{S_{F G}}}+D_{u c_{S_{G H}}}=5+0+5=10$, and $P_{c_{r}}=\left(P_{l}-\right.$ $\left.P_{l_{u c}}\right) / P_{l}=(20-10) / 20=0.5$. As for the path $3, W_{p}=$ $W_{S_{A F}}^{\prime \prime}=0.77, P_{l}=D_{S} A F=7, P_{l_{u c}}=D_{u c_{S}}=5$, and $P_{c_{r}}=\left(P_{l}-P_{l_{u c}}\right) / P_{l}=(7-5) / 7=0.29$.

It shows that with the changing of the $\xi$, the $W_{p}$ of the three navigation paths change (Figure 6). Overall, with the decreasing of $\xi$ (i.e., the increasing of $1-\xi$ ), the $W_{p}$ of path 1 is rising, while that of path 2 and 3 are falling, which reveals that with paying more attention to the top-coverage-ratio of the 
Table 2. Modified weights $\left(W_{S_{i j}}^{\prime \prime}\right)$ based on Equation 3.

\begin{tabular}{ccccccccccccc}
\hline \multirow{2}{*}{$S_{i}$} & $S_{j}$ & \multicolumn{10}{c}{$W_{S_{i j}}^{\prime \prime}$} \\
\cline { 2 - 15 } & & $\xi=1$ & 0.9 & 0.8 & 0.7 & 0.6 & 0.5 & 0.4 & 0.3 & 0.2 & 0.1 & 0 \\
\hline$S_{A}$ & $S_{B}$ & 0.8 & 0.82 & 0.84 & 0.86 & 0.88 & 0.9 & 0.92 & 0.94 & 0.96 & 0.98 & 1 \\
$S_{A}$ & $S_{D}$ & 0 & 0.1 & 0.2 & 0.3 & 0.4 & 0.5 & 0.6 & 0.7 & 0.8 & 0.9 & 1 \\
$S_{A}$ & $S_{F}$ & 0.8 & 0.79 & 0.78 & 0.77 & 0.77 & 0.76 & 0.75 & 0.74 & 0.73 & 0.72 & 0.71 \\
$S_{B}$ & $S_{C}$ & 0.4 & 0.4 & 0.4 & 0.4 & 0.4 & 0.4 & 0.4 & 0.4 & 0.4 & 0.4 & 0.4 \\
$S_{C}$ & $S_{H}$ & 1 & 0.96 & 0.93 & 0.89 & 0.85 & 0.81 & 0.78 & 0.74 & 0.7 & 0.66 & 0.63 \\
$S_{D}$ & $S_{E}$ & 0.4 & 0.46 & 0.52 & 0.58 & 0.64 & 0.7 & 0.76 & 0.82 & 0.88 & 0.94 & 1 \\
$S_{E}$ & $S_{H}$ & 0.2 & 0.28 & 0.36 & 0.44 & 0.52 & 0.6 & 0.68 & 0.76 & 0.84 & 0.92 & 1 \\
$S_{F}$ & $S_{G}$ & 0.4 & 0.36 & 0.32 & 0.28 & 0.24 & 0.2 & 0.16 & 0.12 & 0.08 & 0.04 & 0 \\
$S_{G}$ & $S_{H}$ & 1 & 0.96 & 0.93 & 0.89 & 0.85 & 0.81 & 0.78 & 0.74 & 0.70 & 0.66 & 0.63 \\
\hline
\end{tabular}

Table 3. Three different navigation paths.

\begin{tabular}{|c|c|c|c|c|c|c|c|c|c|c|c|c|}
\hline \multirow{2}{*}{$1-\xi$} & \multicolumn{4}{|c|}{ Path 1} & \multicolumn{4}{|c|}{ Path 2} & \multicolumn{4}{|c|}{ Path 3} \\
\hline & $W_{p}$ & $P_{l}$ & $P_{l_{u c}}$ & $P_{c_{r}}$ & $W_{p}$ & $P_{l}$ & $P_{l_{u c}}$ & $P_{c_{r}}$ & $W_{p}$ & $P_{l}$ & $P_{l_{u c}}$ & $P_{c_{r}}$ \\
\hline 0 & 0.6 & 12 & 12 & 0 & 2.2 & 20 & 10 & 0.5 & 0.8 & 7 & 5 & 0.29 \\
\hline 0.1 & 0.84 & 12 & 12 & 0 & 2.11 & 20 & 10 & 0.5 & 0.79 & 7 & 5 & 0.29 \\
\hline 0.2 & 1.08 & 12 & 12 & 0 & 2.03 & 20 & 10 & 0.5 & 0.78 & 7 & 5 & 0.29 \\
\hline 0.3 & 1.32 & 12 & 12 & 0 & 1.94 & 20 & 10 & 0.5 & 0.77 & 7 & 5 & 0.29 \\
\hline 0.4 & 1.56 & 12 & 12 & 0 & 1.86 & 20 & 10 & 0.5 & 0.77 & 7 & 5 & 0.29 \\
\hline 0.5 & 1.8 & 12 & 12 & 0 & 1.77 & 20 & 10 & 0.5 & 0.76 & 7 & 5 & 0.29 \\
\hline 0.6 & 2.04 & 12 & 12 & 0 & 1.68 & 20 & 10 & 0.5 & 0.75 & 7 & 5 & 0.29 \\
\hline 0.7 & 2.28 & 12 & 12 & 0 & 1.6 & 20 & 10 & 0.5 & 0.74 & 7 & 5 & 0.29 \\
\hline 0.8 & 2.52 & 12 & 12 & 0 & 1.51 & 20 & 10 & 0.5 & 0.73 & 7 & 5 & 0.29 \\
\hline 0.9 & 2.76 & 12 & 12 & 0 & 1.43 & 20 & 10 & 0.5 & 0.72 & 7 & 5 & 0.29 \\
\hline 1 & 3 & 12 & 12 & 0 & 1.34 & 20 & 10 & 0.5 & 0.71 & 7 & 5 & 0.29 \\
\hline
\end{tabular}

path, the traditional shortest path becomes less attractive. That is, if $\xi$ is less than 0.5 , the path 2 is recommended, otherwise, the path 3.

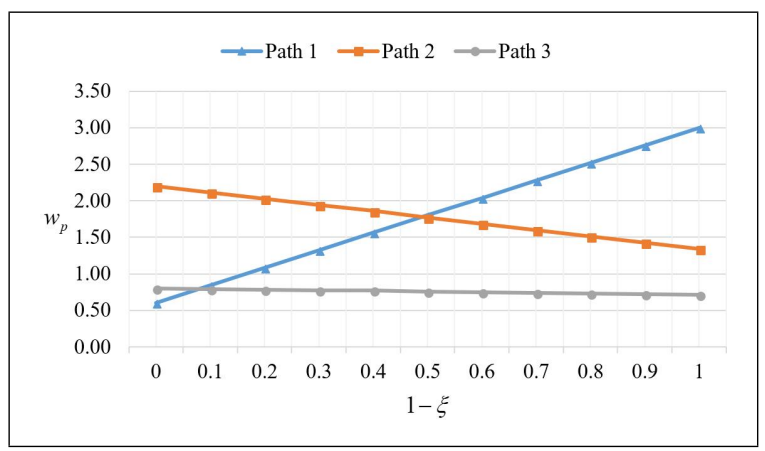

Figure 6. The changes of $W_{p}$ with the changing of the $\xi$.

Comparing paths 1 and 2 , before $\xi$ reaching to 0.4 , the path 2 is recommended for users. For instance, if $\xi=0.4$, The uncovered distance of path $2\left(P_{l_{u c 2}}=12\right)$ is shorter than that of path $1\left(P_{l_{u c 1}}=14\right)$, although its $W_{p} 2$ is smaller than that of path 1 , and the top-coverage-ratio $\left(P_{c_{r 2}}=0\right)$ is smaller than that of path $1\left(P_{c_{r 1}}=0.3\right)$. For this case, NSI-path is computed. But, path 2 is still be recommended, because the length of NSI-path is longer than that of path 2.

In contrast, comparing the path 2 and path 3 , we can find that if $\xi$ less than to 0.5 , the path 2 is recommended, otherwise, the recommendation path is path 3 . Because when the $\xi>0.5$, the $W_{p}$ of path 3 smaller than that of path 2. Meanwhile, the uncovered distance of path 3 is shorter that than of path 2, and the top-coverage-ratio changes larger than that of path 2 .

Comparing paths 1 and 3 only, at the beginning (i.e., $\xi=1$ ), the two paths are the same from the $W_{p}$ aspect. But, with the decreasing of $(\xi)$, path 3 becomes the recommended path considering its $W_{p}$ becomes always less than that of path 1 . Meanwhile, its covered distance is longer than that of path 1 $\left(P_{l_{c} 3}>P_{l_{c} 1}\right)$ and top-coverage-ratio is bigger than that of path $1\left(P_{c_{r 3}}>P_{c_{r 1}}\right)$.

\section{IMPLEMENTATION}

\subsection{Agent \& Test Area}

The agent in this implementation is a pedestrian, which is modelled as a 3D object with length $(625 \mathrm{~mm} \leq 1 \leq 875 \mathrm{~mm})$, width ( $\mathrm{w} \geq 375 \mathrm{~mm}$ ), and height $(\mathrm{h} \geq 1930 \mathrm{~mm}$ ) (Yan et al., 2019b).

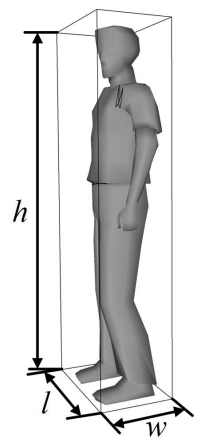

Figure 7. A pedestrian is modelled as a 3D object.

The test area is part of the university campus in UNSW (Figure 8). There are seven types of objects, including buildings, shelters, roads, green areas, hand railings, enclosing walls, and fences. The buildings indicate I-spaces and act as physical boundaries. Hand railings, enclosing walls, and fences also 
serve as physical boundaries. Shelters represent areas of sI-spaces while roads and green areas are sO-spaces and O-spaces.

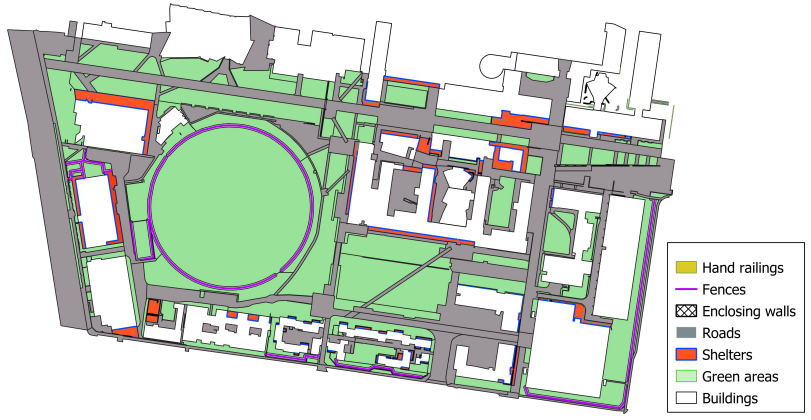

Figure 8 . Selected area of university campus for testing.

The data of the indoor is a Building Information Modelling (BIM) model. The initial data of semi-indoor, semi-outdoor, and outdoor is a 2D map. Then the footprints in the 2D map are manually corrected to be topologically correct.

\subsection{D Spaces \& Navigation Network Derivation}

Only the sI-spaces formed by the building with the BIM model are reconstructed based on the approaches presented in (Yan et al., 2018, Yan et al., 2019b). The process of reconstructing other sI-spaces formed by shelters is extruding footprints of shelters to a certain height, which is similar to the approach for sO-spaces and O-spaces presented in (Yan et al., 2020). Here, a certain height is employed for sI-spaces, sO-spaces, and $\mathrm{O}$-spaces reconstruction. This paper assigns a certain height (2 meters) for sI-spaces, sO-spaces, and O-spaces reconstruction, because we consider this height is sufficient for pedestrian navigation. All spaces in the test area are shown in Figure 9.

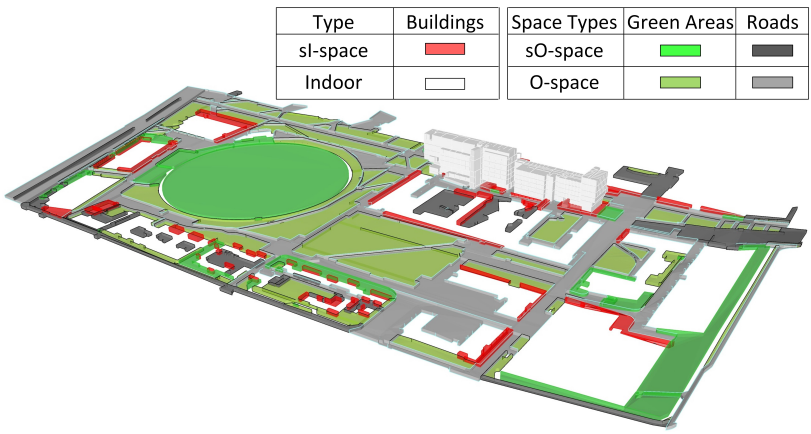

Figure 9. BIM spaces and created 3D spaces in the test area.

Theoretically, the navigation network can be derived from 3D spaces on the basis of Poincare duality (Munkres, 1984). Indoor spaces are generally connected by doors. Thus, the process is extracting room (space) as nodes and connected by a node extracted from Door (space). However, semi-indoor, semi-outdoor, and outdoor spaces are naturally connected by virtual boundaries rather than doors. Therefore, we use additional vertex on the face two spaces touch to indicate how to traverse the spaces. Even if the path does not intersect the spaces, the vertices are still needed, see the abstracted example in Figure 4(c). Thus, curve edges and extra vertices are employed to force the navigation links to pass through the sharing of virtual boundaries.

All the indoor cases without BIM models are omitted in this experiment, because we used building footprints as physical boundaries, which makes such I-spaces become isolated to other any spaces. The derived navigation network is shown in Figure 10.

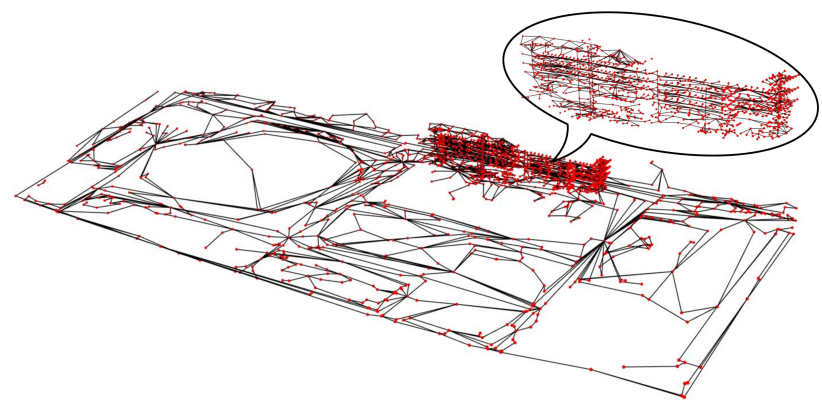

Figure 10. The navigation network automatically derived from 3D spaces based on Poincaré duality.

\subsection{Path planning}

The MTC-path and NSI-path are implemented with two navigation cases: $\mathrm{A} \rightsquigarrow \mathrm{B}, \mathrm{C} \rightsquigarrow \mathrm{D}$. For comparison, OSM and Google Maps are also used for path planning. Rhinoceros (with Grasshopper) is used for conducting the whole process and visualizing the results.

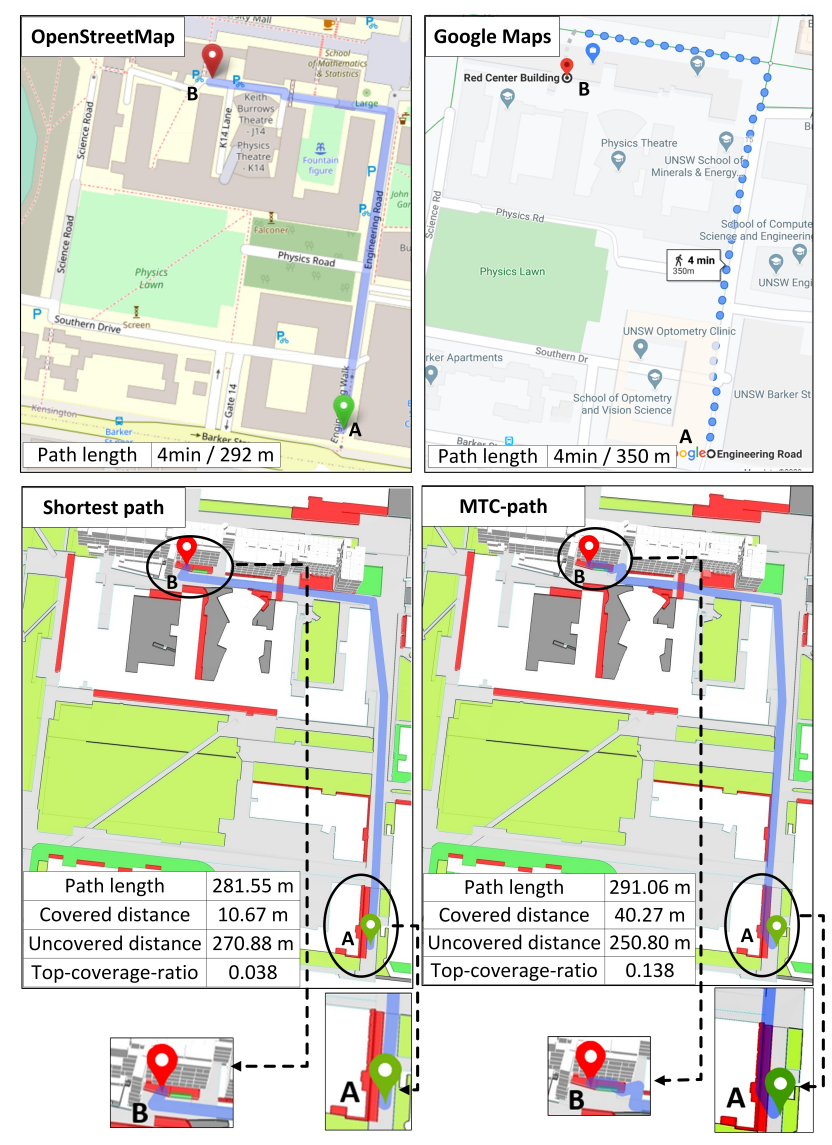

Figure 11. Navigation paths of A $\rightsquigarrow$ B. Differences between shortest path and MTC-path are marked by black ellipses, which shows where the sI-spaces are involved.

The first navigation case takes $\mathrm{A}$ as departure and $\mathrm{B}$ as the destination. The shortest path and MTC-path can be seen in Figure 11. All three different navigation systems can offer the shortest path, but only our approach can offer the MTC-path and NSI-path. The navigation path computed by Google Maps 

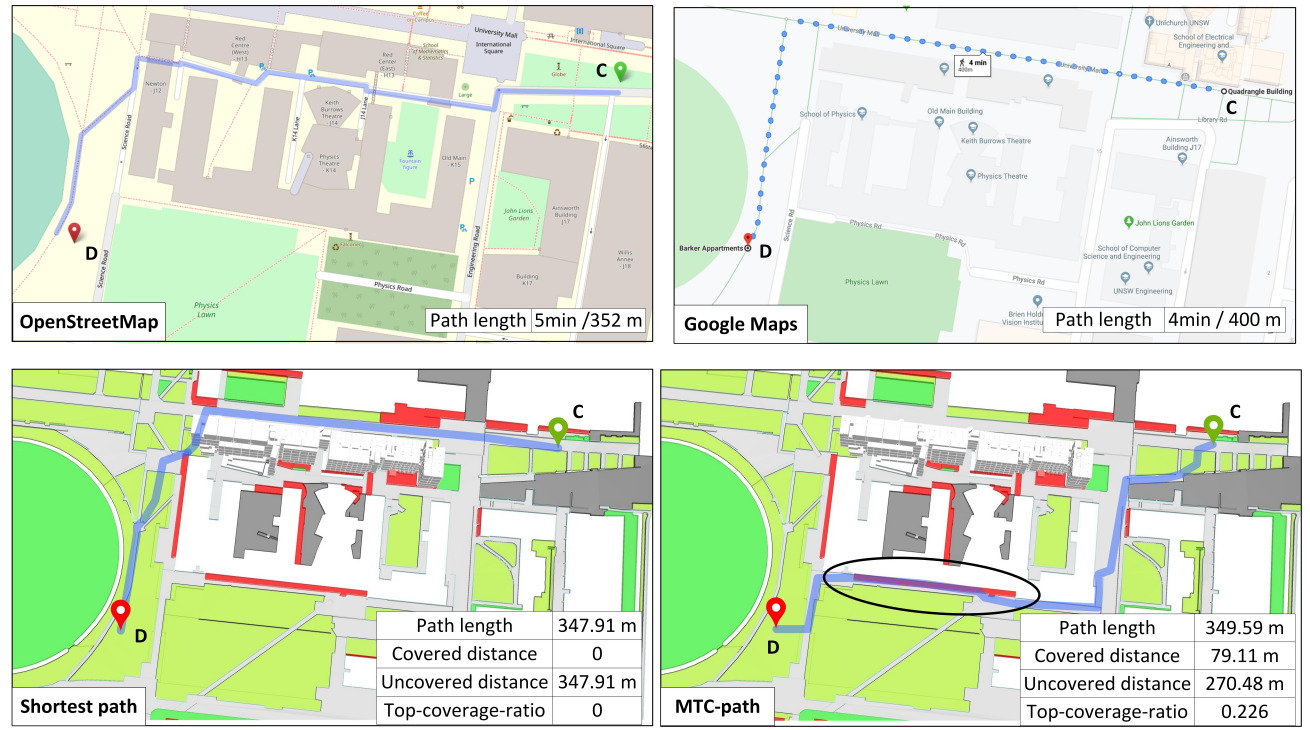

Figure 12. Navigation paths of $\mathrm{C} \rightsquigarrow \mathrm{D}$. The part marked by black ellipse shows where the sI-spaces are involved in MTC-path.

Table 4. Comparisons of three navigation systems. $\times$ means no while $\checkmark$ means yes.

\begin{tabular}{cccccc}
\hline Approach & sI-space & 2D/3D & Shortest path & MTC-path & NSI-path \\
\hline OSM & $\times$ & 2D & $\checkmark$ & $\times$ & $\times$ \\
Google Maps & $\times$ & 2D & $\checkmark$ & $\times$ & $\times$ \\
Our approach & $\checkmark$ & 3D & $\checkmark$ & $\checkmark$ & $\checkmark$ \\
\hline
\end{tabular}

obviously shows this system tries to guide pedestrians to go along with roads. Furthermore, the third dimension (vertical constraint) of outdoor spaces in OSM and Google Maps is neglected, but it is considered in our approach, because the navigation network of our approach is derived from 3D spaces.

Our approach not only can offer different path options but also detailed information of the paths, such as covered/uncovered distance as well as the top-coverage-ratio. The MTC-path in Figure 11 includes as many sI-spaces as possible to increase the top-coverage-ratio, see the black circled part.

The second navigation case is from $\mathrm{C}$ (departure) to $\mathrm{D}$ (destination) (Figure 12). The paths of this case vary greatly. The path offered by OSM shows the pedestrians can go through a building. Navigation in Google Maps still tried to guide pedestrians to go along with roads. We expect our approach to have a similar shortest path to the result from OSM, but with the reason that this building does not have a BIM model and therefore is excluded, the shortest path of our approach slightly changes. In the MTC-path, it clearly shows that it tries to use sI-spaces to increase the covered distance and top-coverage-ratio.

Based on the path selection strategy, both MTC-path in the two cases is recommended, since the uncovered distances of them are shorter than that of their corresponding shortest path ( 250.80 vs 270.88 , and 270.48 vs 347.91 ) and the coverage ratio of the two MTC-paths is larger than that of their shortest paths ( 0.138 vs 0.038 , and 0.226 vs 0 ). As mentioned above, the NSI-path is a compromise option when neither the shortest path nor MTC-path is not recommended. Due to both MTC-path in two cases is recommended, the NSI-path of the two navigation cases is not computed, but it is for sure, the NSI-path can be planned with our approach.

We compare the path planning results of OSM, Google Maps, and our approach from five aspects (Table 4): (i) if the sI-space is considered in navigation map, (ii) navigation performs in 2D or $3 \mathrm{D}$, (iii) if the shortest path can be computed, (iv) if it can compute the MTC-path, and (v) if it can provide NSI-path.

The results show that current navigation applications do not consider sI-spaces in navigation map, perform navigation in $2 \mathrm{D}$, and offer the shortest path as the only path option. In comparison, the navigation paths show that our approach performs navigation in 3D. More importantly, it not only can offer the shortest path but also the MTC-path and NSI-path.

\section{CONCLUSION AND FUTURE WORK}

This paper presents two path options based on sI-spaces, including: MTC-path and NSI-path. Both paths are developed to meet the user's pursuit of protection from the top direction during their navigation. This research has three contributions to navigation path planning: (a) sI-spaces are included in navigation paths as destination or departure; (b) MTC-path and NSI-path are computed for users who need the shortest path with as many covers from the top as possible; (c) The navigation performs in $3 \mathrm{D}$, which aligns with the real situation that navigation is a process of pedestrians move from one $3 \mathrm{D}$ space to another.

In the future, we will extend this research to new path options with sI-spaces to I-spaces, even sO-spaces or O-spaces for other navigation preferences. For instance, a path with as many I-spaces as possible could be attractive in hot summer, since the I-spaces are generally equipped with air conditioners. In short, it is possible to develop multiple path options based on semantics of spaces and preferences of users.

It should be mentioned that the 3D spaces are one of the main limitations of this research - that are further utilized for 
navigation network derivation - in which sI-spaces are correctly reconstructed. Therefore, more aspects that are related to sI-spaces need to be investigated in depth. For example, setting the threshold of $C^{T}$ for sI-space selection is a precondition of using sI-spaces for MTC-path and NSI-path. In this paper, the threshold is set as 0.8 because the authors consider a top with a $C^{T} \geq 0.8$ is enough for a pedestrian to escape from the strong sun. If pedestrians desire to use the tops to escape from the rain, the threshold of $C^{T}$ for sI-space could be set as 0.95 .

Furthermore, it is necessary to investigate the preferences of users, which would have a higher impact on their decisions compared to time or distance-based routes that are currently in use (shortest distance/time path). This approach is expected to be combined with different attributes or challenges, and various metrics such as the number of turns, and the number of decision points, among others.

\section{ACKNOWLEDGEMENTS}

This work is supported by the program of China Scholarships Council (No.201606410054). The authors would like to thank Ordnance Survey GB (https://www.ordnancesurvey.co.uk) and 1Spatial (https://1spatial.com/) for sponsoring the publication of this paper.

\section{REFERENCES}

Alam, MS, Perugu, H, McNabola, A, 2018. A comparison of route-choice navigation across air pollution exposure, $\mathrm{CO} 2$ emission and traditional travel cost factors. Transportation Research Part D: Transport and Environment, 65, 82-100.

Andreev, Simeon, Dibbelt, Julian, Nöllenburg, Martin, Pajor, Thomas, Wagner, Dorothea, 2015. Towards realistic pedestrian route planning. 15th Workshop on Algorithmic Approaches for Transportation Modelling, Optimization, and Systems (ATMOS 2015), Schloss Dagstuhl-Leibniz-Zentrum fuer Informatik.

Balata, Jan, Berka, Jakub, Mikovec, Zdenek, 2018. Indoor-outdoor intermodal sidewalk-based navigation instructions for pedestrians with visual impairments. International Conference on Computers Helping People with Special Needs, Springer, 292-301.

Cambra, Paulo Jorge, Gonçalves, Alexandre, Moura, Filipe, 2019. THE DIGITAL PEDESTRIAN NETWORK IN COMPLEX URBAN CONTEXTS: A PRIMER DISCUSSION ON TYPOLOGICAL SPECIFICATIONS. Finisterra, 54, 155-170.

Dijkstra, E.W., 1959. A note on two problems in connexion with graphs. Numerische Mathematik, 1, 269-271.

Duckham, Matt, Kulik, Lars, 2003. "simplest" paths: automated route selection for navigation. International Conference on Spatial Information Theory, Springer, 169-185.

Dudas, Patrick M, Ghafourian, Mahsa, Karimi, Hassan A, 2009. Onalin: Ontology and algorithm for indoor routing. 2009 Tenth International Conference on Mobile Data Management: Systems, Services and Middleware, IEEE, 720-725.

Ghafourian, Mahsa, Karimi, Hassan A, 2009. Cad/gis integration issues for seamless navigation between indoor and outdoor environments. CAD and GIS Integration, Auerbach Publications, 141-154.
Golledge, Reginald G, 1995. Path selection and route preference in human navigation: A progress report. International conference on spatial information theory, Springer, 207-222.

Howard, Charlene, Burns, Elizabeth K, 2001. Cycling to work in Phoenix: Route choice, travel behavior, and commuter characteristics. Transportation Research Record, 1773, 39-46.

Karimi, Hassan A, Jiang, Ming, Zhu, Rui, 2013. Pedestrian navigation services: challenges and current trends. Geomatica, 67, 259-271.

Kimmel, Ron, Amir, Arnon, Bruckstein, Alfred M., 1995. Finding shortest paths on surfaces using level sets propagation. IEEE Transactions on Pattern Analysis and Machine Intelligence, 17, 635-640.

Liu, Liu, Zlatanova, Sisi, 2013. A two-level path-finding strategy for indoor navigation. Intelligent systems for crisis management, Springer, 31-42.

Millonig, Alexandra, Schechtner, Katja, 2007. Developing landmark-based pedestrian-navigation systems. IEEE Transactions on Intelligent Transportation Systems, 8, 43-49.

Munkres, James R, 1984. Elements of algebraic topology. 2, Addison-Wesley Menlo Park.

Sharker, Monir H, Karimi, Hassan A, Zgibor, Janice C, 2012. Health-optimal routing in pedestrian navigation services. Proceedings of the First ACM SIGSPATIAL International Workshop on Use of GIS in Public Health, ACM, 1-10.

Wang, Zhiyong, Zlatanova, Sisi, 2019. Safe Route Determination for First Responders in the Presence of Moving Obstacles. IEEE Transactions on Intelligent Transportations Systems, 1-19.

Yan, Jinjin, Diakité, Abdoulaye A, Zlatanova, Sisi, 2018. AN EXTRACTION APPROACH OF THE TOP-BOUNDED SPACE FORMED BY BUILDINGS FOR PEDESTRIAN NAVIGATION. ISPRS Annals of Photogrammetry, Remote Sensing \& Spatial Information Sciences, 4, 247-254.

Yan, Jinjin, Diakité, Abdoulaye A, Zlatanova, Sisi, 2019a. A generic space definition framework to support seamless indoor/outdoor navigation systems. Transactions in GIS, 23, 1273-1295.

Yan, Jinjin, Diakité, Abdoulaye A, Zlatanova, Sisi, Aleksandrov, Mitko, 2019b. Top-Bounded Spaces Formed by the Built Environment for Navigation Systems. ISPRS International Journal of Geo-Information, 8, 224.

Yan, Jinjin, Diakité, Abdoulaye A, Zlatanova, Sisi, Aleksandrov, Mitko, 2020. Finding outdoor boundaries for 3D space-based navigation. Transactions in GIS, 24, 371-389.

Zar, Myat Thu, Sein, Myint Myint, 2016. Finding shortest path and transit nodes in public transportation system. Genetic and Evolutionary Computing, Springer, 339-348.

Zhu, Qing, Li, Yun, Xiong, Qing, Zlatanova, Sisi, Ding, Yulin, Zhang, Yeting, Zhou, Yan, 2016. Indoor multi-dimensional location gml and its application for ubiquitous indoor location services. ISPRS International Journal of Geo-Information, 5, 220 . 